\title{
CHSH inequality: Quantum probabilities as classical conditional probabilities
}

\author{
Andrei Khrennikov \\ International Center for Mathematical Modeling \\ in Physics and Cognitive Sciences \\ Linnaeus University, Växjö-Kalmar, Sweden
}

\begin{abstract}
The celebrating theorem of A. Fine implies that the CHSH inequality is violated if and only if the joint probability distribution for the quadruples of observables involved the EPR-Bohm-Bell experiment does not exist, i.e., it is impossible to use the classical probabilistic model (Kolmogorov, 1933). In this note we demonstrate that, in spite of Fine's theorem, the results of observations in the EPRBohm-Bell experiment can be described in the classical probabilistic framework. However, the "quantum probabilities" have to be interpreted as conditional probabilities, where conditioning is with respect to fixed experimental settings. Our approach is based on the complete account of randomness involved in the experiment. The crucial point is that randomness of selections of experimental settings has to be taken into account. This approach can be applied to any complex experiment in which statistical data are collected for various (in general incompatible) experimental settings. Finally, we emphasize that our construction of the classical probability space for the EPR-Bohm-Bell experiment cannot be used to support the hidden variable approach to the quantum phenomena. The classical random parameter $\omega$ involved in our considerations cannot be identified with the hidden variable $\lambda$ which is used the Bell-type considerations.
\end{abstract}

\section{Introduction}

Although this year we celebrate the 50th anniversary of Bell's inequality [1] (see also [2]), its interpretations and, in particular, interpretations of its probabilistic structure are still the hot topic of 
discussions on foundations of quantum mechanics and quantum information theory, e.g., in [3]-11. Since Bell's types inequalities play the fundamental role in various applications of quantum information, especially in quantum cryptography and theory of quantum random generators, the debates on the Bell inequality have important consequences for justification of modern quantum technologies (see again, e.g., [3]-[11]). We also remark that recently essential progress was approached in the performance of a loophole free Bell test [12, [13] (see also [14]), although it is still unclear when such a final test will be finally performed.

There is the common opinion that violation of the Bell-type inequalities by quantum correlations implies that the laws of classical probability theory (based on the Kolmogorov measure-theoretic axiomatics [15], 1933) cannot be applied to the description of quantum phenomena (at least for entangled systems). The heuristic roots of such a viewpoint are clear, cf. [16]-[31. The statistical data used to violate the Bell-type inequalities are collected for pairs of incompatible experimental settings. Since in the Kolmogorov model all observables have to be represented by random variables (measurable functions) on the same sample space, it is reasonable to expect that such a construction cannot be used in the case of incompatibility, see, e.g., Such heuristic reasoning can be mathematically justified with aid the celebrating theorem of A. Fine [32]. This theorem states that the CHSHinequality [33] is violated if and only if the joint probability distribution for the quadruples of observables involved the EPR-Bohm-Bell experiment does not exist, i.e., it is impossible to use the classical probabilistic model (Kolmogorov, 1933).

In this note we demonstrate that, in spite of the Fine theorem the results of observations in the EPR-Bohm-Bell experiment can be described in the classical probabilistic framework. However, the "quantum probabilities" have to be interpreted as conditional probabilities, where conditioning is with respect to fixed experimental settings. Our approach is based on the complete account of randomness involved in the experiment. The crucial point is that randomness of selections of experimental settings has to be taken into account. In this paper we present the general Kolmogorovian construction for complex experiments combing a few (in general incompatible) experimental settings; the concrete model of the Kolmogorov space was presented in [34], 35. We emphasize that our construction can be used for data of any kind, i.e., not only for data from quantum experiments [36], cf. also with the recent papers of Dzhafarov and Kujala [37, 38]. We point out that the problem of embedding of quantum statistical data into the classical probabilistic model has interesting couplings to the 
problem of freewill [35], cf. with the paper of Kofler et al. 39].

We remark that the problem of inter-relation between quantum and classical probability was studied in hundreds of papers. Here we even do not try to present the corresponding bibliography, see, e.g., [29], [27] for reviews; besides of cited papers related to the Bell inequality, we can mention the works of De Gosson, e.g., [40], [41] on inter-relation between probabilistic structures of classical and quantum mechanics and a series of papers of Manko et al., see, e.g., [42], [43, on the classical probabilistic representation of QM; see also the article of D'Ariano [44] on the information characterization of the quantum probabilistic structure.

Finally, we emphasize that our construction of the classical probability space for the EPR-Bohm-Bell experiment cannot be used to support the hidden variable approach to the quantum phenomena. The classical random parameter $\omega$ involved in our considerations cannot be identified with the hidden variable $\lambda$ which is used the Belltype considerations. Randomness of $\omega$ is not reduced to randomness of the state preparation; it also includes randomness of selections of experimental settings. The model is so tricky that these randomnesses cannot be separated from each other. Therefore we are not able to determine (even probabilistically) the values of incompatible physical observables on the basis of "hidden variables" associated with prepared systems.

Nevertheless, this work made smaller the gap between classical and quantum probabilities by interpreting the latter as classical conditional probabilities.

\section{Experiments on compound systems combining a few experimental settings}

There is a source producing compound systems; we consider the simplest case of systems consisting of two subsystems, $S=\left(S_{1}, S_{2}\right)$. There are given two measurement devices labeled as the $A$-device and the $B$-device; the first one performs measurements on $S_{1}$ and the second on $S_{2}$. It can (but need not) be assumed that the devices are spatially separated for sufficiently large distance. Each device can measure a few observables, $A_{i}, i=1, \ldots, n, B_{j}, j=1, \ldots, m$. Again for simplicity we restrict the number of observables per device to two, $n=m=2$, and consider observables taking values \pm 1 . (A bit later the range of values will be extended to include the event of nondetection.) At the fixed moment of time each device can measure only one observable. Selections of observables to be measured are orchestrated by 
random generators denoted by $a$ and $b$. In our case each takes two values $a, b=1,2$ determining devices' settings corresponding to measurements of observables labeled by these indexes.

Measurement of each observable is performed with the aid of a pair of detectors, $D_{i}^{A}(-), D_{i}^{A}(+)$ and $D_{i}^{B}(-), D_{i}^{B}(+), i=1,2$. At "each side" the corresponding random generator controls the flow of (sub)systems in the following way. To be concrete consider the $A$ device. If the random generator $a$ takes the value $a=1$, then the subsystem $S_{1}$ in the pair $S$ arrives to one of the detectors $D_{1}^{A}(-), D_{1}^{A}(+)$ and produces a click. (It is assumed that all detectors have 100\% efficiency.) For this value of $a$, there is no way for $S_{1}$ to arrive to one of the detectors $D_{2}^{A}(-), D_{2}^{A}(+)$. Moreover, there is neither random background, so no one of the detectors $D_{2}^{A}(-), D_{2}^{A}(+)$ can click 11 If $D_{1}^{A}(-)$ clicks, we set $A_{1}=-1$, and if $D_{1}^{A}(+)$ clicks, we set $A_{1}=+1$. In this context, $D_{2}^{A}( \pm)$ detectors do not click. We label this situation for the $A_{2}$-observable by setting $A_{2}=0$; thus nondetection is labeled by zero. This is a trivial, but important point: in our model the range of values of observables is extended by adding the value (zero) corresponding to nondetection.

Typically in quantum experiments the number of detectors per device is restricted to just one pair, e.g., the same pair of detectors is used to measure both $A_{1}$ and $A_{2}$. Our model also covers this case by identification the detectors for $i=1,2$. However, even in such a case it is useful to preserve the labeling by the experimental setting. In principle, change of experimental setting can modify functioning of detectors.

This is the most general description of experiments on compound systems combing selections of a few experimental settings. This scheme can be applied both to classical and quantum systems. In quantum optics settings are given by angles determining orientations of PBSs, $\theta_{1}, \theta_{2}$ for the $A$-device and $\theta_{1}^{\prime}, \theta_{2}^{\prime}$ for the $B$-device. We can also consider an experiment (classical or quantum) in which one of observables is position and another momentum.

The main message of this section: if a few settings of measurement devices are combined in one experiment, then the rule of their combi-

\footnotetext{
${ }^{1}$ As a possible realization, we can consider the following experimental framework. The random generator $a$ is coupled to a block which splits the channel going from the source of photons in the $A$-direction into two channels, which are also labeled by $i=1,2$. Each of the channels is coupled to its own polarization beam splitter (PBS) which has the fixed orientation given by the angle $\theta_{i}$ and the PBS in the $i$ th channel is coupled to its pair of the detectors $D_{i}^{A}(-)$, polarization down, and $D_{i}^{A}(+)$, polarization up. Thus at each side there are two PBSs (corresponding to the fixed orientations) and totally 4 detectors. The complete two-side experimental scheme is based on 4 PBSs and 8 detectors.
} 
nation has to be present in the corresponding probabilistic model; in our model this rule is given by the pair of random generators.

\section{Classical probability model describ- ing combination of a few experimental settings}

Let $(\Omega, \mathcal{F}, p)$ be Kolmogorov probability space. Consider four random variables $A_{i} \equiv A_{i}(\omega)$ and $B_{i} \equiv B_{i}(\omega), i=1,2$, which yield the values $\{-1,0,+1\}$. (They describe observables.) We also assume that on the same probability space there are defined other two random variables $a \equiv a(\omega)$ and $b \equiv b(\omega)$; they yield the values $i=1,2$. (They describe random generators.)

These random variables are connected by the following conditions $(i=1,2)$ :

$$
\begin{aligned}
& \text { C1 }: p\left(A_{i}=0 \mid a \neq i\right)=1, p\left(B_{i}=0 \mid b \neq i\right)=1 ; \\
& \text { C2 }: p\left(A_{i}=0 \mid a=i\right)=0, p\left(B_{i}=0 \mid b=i\right)=0 .
\end{aligned}
$$

Thus $A_{i}$ and $B_{i}$ can take nonzero values only under conditions $a=i$ and $b=i$, respectively; e.g.,

$$
\begin{aligned}
& p\left(A_{i}=-1 \mid a=i\right)+p\left(A_{i}=+1 \mid a=i\right)=1-p\left(A_{i}=0 \mid a=i\right)=1 \\
& p\left(A_{i}=-1 \mid a \neq i\right)+p\left(A_{i}=+1 \mid a \neq i\right)=1-p\left(A_{i}=0 \mid a \neq i\right)=0 .
\end{aligned}
$$

These conditions have different physical meanings. By C1 there is no random background. For example, for the $A$-device, detectors in a pair coupled to the $i$-setting cannot click if systems are sent to the pair corresponding to another setting. By C2 all detectors have $100 \%$ efficiency. Thus to model less efficient detectors, one has to proceed without C2. (Our model can be easily modified to include both the presence of random background and usage of detectors with the efficiency less than $100 \%$. However, we want to present the essentials of our approach for embedding quantum probabilities into the classical probability model; we do not want to shadow the essentials by "technicalities".)

By using these conditions we derive (with the aid of the formula of total probability and more generally additivity of probability) some properties of unconditional probabilities.

First of all we have $(i=1,2$,$) :$

$p\left(A_{i}=0\right)=p(a=i) p\left(A_{i}=0 \mid a=i\right)+p(a \neq i) p\left(A_{i}=0 \mid a \neq i\right)=p(a \neq i)$. 
Thus the probability that the detectors $D_{i}^{A}( \pm)$ do not click equals to the probability of non-selection of the $i$-setting. In the same way we obtain

$$
p\left(B_{i}=0\right)=p(b \neq i) .
$$

By using (44) we find that, for $\epsilon, \epsilon^{\prime}= \pm 1$,

$$
p\left(A_{i}=\epsilon\right)=p(a=i) p\left(A_{i}=\epsilon \mid a=i\right)
$$

or another way around

$$
p\left(A_{i}=\epsilon \mid a=i\right)=\frac{1}{p(a=i)} p\left(A_{i}=\epsilon\right) .
$$

As the next step we find the joint probability distribution for the pairs of the random variables $\left(A_{i}, B_{j}\right)$. Set $\epsilon, \epsilon^{\prime}= \pm 1$. Then

$$
p\left(A_{i}=\epsilon, B_{j}=\epsilon^{\prime}\right)=\sum_{k, m=1,2} p\left(A_{i}=\epsilon, B_{j}=\epsilon^{\prime}, a=k, b=m\right) .
$$

Now suppose that, e.g., $k \neq i$. Then we obtain

$p\left(A_{i}=\epsilon, B_{j}=\epsilon^{\prime}, a=k, b=m\right) \leq p\left(A_{i}=\epsilon, a=k\right)=p(a \neq i) p\left(A_{i}=\epsilon \mid a \neq i\right)=0$.

Thus only the terms with $k=i$ and $m=j$ give nontrivial contributions into the sum. We obtain the following formula:

$$
p\left(A_{i}=\epsilon, B_{j}=\epsilon^{\prime}\right)=p\left(A_{i}=\epsilon, B_{j}=\epsilon^{\prime} \mid a=i, b=j\right) p(a=i, b=j)
$$

or

$$
p\left(A_{i}=\epsilon, B_{j}=\epsilon^{\prime} \mid a=i, b=j\right)=\frac{1}{p(a=i, b=j)} p\left(A_{i}=\epsilon, B_{j}=\epsilon^{\prime}\right) .
$$

Now we find joint probabilities of non-detection:

$$
\begin{gathered}
p\left(A_{i}=0, B_{j}=0\right)=\sum_{k, m=1,2} p\left(A_{i}=0, B_{j}=0, a=k, b=m\right) \\
=p\left(A_{i}=0, B_{j}=0, a \neq i, b \neq j\right) .
\end{gathered}
$$

Hence,

$$
p\left(A_{i}=0, B_{j}=0\right)=p\left(A_{i}=0, B_{j}=0 \mid a \neq i, b \neq j\right) p(a \neq i, b \neq j) .
$$

We can also rewrite (12) in the following way

$$
p\left(a \neq i, b \neq j \mid A_{i}=0, B_{j}=0\right)=1 .
$$


No detection for the pair of observables $\left(A_{i}, B_{j}\right)$ implies that the pair $(i, j)$ of setting was not selected. In the same way we derive that (for $\left.\epsilon, \epsilon^{\prime}= \pm 1\right)$

$$
\begin{gathered}
p\left(A_{i}=\epsilon, B_{j}=0\right)=p\left(A_{i}=\epsilon, B_{j}=0 \mid a=i, b \neq j\right) p(a=i, b \neq j) \\
p\left(A_{i}=0, B_{j}=\epsilon^{\prime}\right)=p\left(A_{i}=0, B_{j}=\epsilon^{\prime} \mid a \neq i, b=j\right) p(a \neq i, b=j) .
\end{gathered}
$$

\subsection{Quantum probabilities as conditional prob- abilities}

Now this is the time to explain why we are interested in coupling between "absolute probabilities" and conditional probabilities. "Absolute probabilities" contain the contribution of randomness involved in selection of the experimental settings. This randomness is not present in conditional probabilities. Therefore the conditional probabilities match with the probabilities which are obtained by experimenters for the fixed experimental settings. If we forget (at least for a moment) about non-detection probabilities, then the basic relation of this paper is given by the formula (111).

In particular, consider experiments to test the Bell-type inequalities. Here the probabilities $p\left(A_{i}=\epsilon, B_{j}=\epsilon^{\prime} \mid a=i, b=j\right)$ correspond to probabilities obtained for the fixed pair of angles $\left(\theta_{i}, \theta_{j}^{\prime}\right)$. Theoretically these probabilities are predicted by the formalism of quantum mechanics. The relation (11) implies the following interpretation of quantum probabilities: from the viewpoint of the classical probabilistic model these are conditional probabilities with respect to selections of pairs of experimental settings. Of course, features of conditional probabilities do not coincide with features of absolute probabilities. Therefore attempts of interpretation of quantum probabilities as "absolute probabilities** induce problems and even paradoxes. We shall consider this problem closer in section 7.

\section{Counterfactuals}

As in any classical probabilistic model, in our model there are well defined not only joint probability distributions for the pairs of random variables $\left(A_{i}, B_{j}\right)$, but even for the pairs $\left(A_{i}, A_{j}\right)$ and $\left(B_{i}, B_{j}\right)$. In quantum mechanics the corresponding observables are incompatible and cannot be measured jointly. It seems that there is a kind of contradiction. However, this is not the case. In our model of experiment (section 2), e.g., observables $A_{1}$ and $A_{2}$ cannot be measured jointly, 
although the corresponding pair of random variables is well defined, $\omega \rightarrow\left(A_{1}(\omega), A_{2}(\omega)\right.$, for $\omega \in \Omega$. It is easy to see that, for $\epsilon_{1}, \epsilon_{2}= \pm 1$,

$$
p\left(A_{1}=\epsilon_{1}, A_{2}=\epsilon_{2}\right)=0 .
$$

In other words the measure of the set

$$
O_{A}\left(\epsilon_{1} \epsilon_{2}\right)=\left\{\omega \in \Omega: A_{1}(\omega)=\epsilon_{1}, A_{2}(\omega)=\epsilon_{2}\right\}
$$

equals to zero. One can say that in our model of experiment counterfactuals exist, but the probability to meet them equals to zero.

To prove (17), we represent its left-hand side as

$$
\begin{gathered}
p\left(A_{1}=\epsilon_{1}, A_{2}=\epsilon_{2}\right)=p\left(A_{1}=\epsilon_{1}, A_{2}=\epsilon_{2}, a=1\right)+p\left(A_{1}=\epsilon_{1}, A_{2}=\epsilon_{2}, a=2\right) \\
\leq p\left(A_{2}=\epsilon_{2}, a=1\right)+p\left(A_{1}=\epsilon_{1}, a=2\right) \\
=p\left(A_{2}=\epsilon_{2} \mid a=1\right) p(a=1)+p\left(A_{1}=\epsilon_{1} \mid a=2\right) p(a=2)=0,
\end{gathered}
$$

where at the last step the equality (4) was applied.

Of course, the same can be said about the probability distribution of quadruples. For $x_{1}, x_{2}, y_{1}, y_{2}=0, \pm 1$, if $x_{1} x_{2} \neq 0$ or $y_{1} y_{2} \neq 0$, then

$$
p\left(A_{1}=x_{1}, A_{2}=x_{2}, B_{1}=y_{1}, B_{2}=y_{2}\right)=0 .
$$

Thus the measure of of the set

$O_{A}\left(x_{1} x_{2} y_{1} y_{2}\right)=\left\{\omega \in \Omega: A_{1}(\omega)=x_{1}, A_{2}(\omega)=x_{2}, B_{1}(\omega)=y_{1}, B_{2}(\omega)=y_{2}\right\}$,

where $x_{1} x_{2} \neq 0$ or $y_{1} y_{2} \neq 0$, equals to zero.

\section{Classical probabilistic description of (non)locality}

We remark that in the Bell framework the notion of (non)locality was not formulated in the space-time picture which is standard for relativity theory. And in this paper we do not plan to discuss this problem, see, e.g., [27], [45]-[47]) for the corresponding formulation and discussion. The original Bell discussion on (non)locality was given in terms of probabilistic independence and in this paper we shall proceed in the same way. In our model Bell-type locality is formalized with the aid of the following conditions on observables (represented by the random variables $\left.A_{i}, B_{j}, i, j=1,2\right)$ and on random generators determining experimental settings (and represented by the random variables $a, b$ ). 
LO The observables are indexed by just one index encoding to selections of the corresponding experimental setting, i.e., $A_{i}, B_{j}$ and not $A_{i j}, B_{i j}$.

LIG The random generators determining selections of the experimental settings are independent random variables:

$$
p(a=i, b=j)=p(a=i) p(b=j)
$$

or

$$
p(a=i \mid b=j)=p(a=i), p(b=j \mid a=i)=p(b=j) .
$$

However, it is not enough to assume that selections of the settings are done independently. One also has to be sure that observables "at one side" are independt from selections of the settings "at another side". This condition jointly with LG leads us to condition:

LIOG The random vectors $\left(A_{i}, a\right), i=1,2$, are independent from the random generator $b$ and the random vectors $\left(B_{i}, b\right), i=1,2$, are independent from the random generator a.

We remark that LIOG trivially implies LIG. Thus we now proceed under conditions LO, LIOG (of course, in combination with C1, C2). In the framework of the Bell-type tests we consider these conditions as classical probabilistic formalization of locality ( mod remark at the beginning of this section); violation of LO or LIOG leads to (probabilistic) nonlocality.

We now analyze consequences of LIOG; for $x=0, \pm 1, m, k=1,2$, we have: $p\left(A_{i}=x, a=k\right)=p\left(A_{i}=x, a=k \mid b=m\right)$. Thus $p\left(A_{i}=\right.$ $x)=\sum_{k=1,2} p\left(A_{i}=x, a=k\right)=\sum_{k=1,2} p\left(A_{i}=x, a=k \mid b=m\right)$. Hence,

$$
p\left(A_{i}=x \mid b=m\right)=p\left(A_{i}=x\right),
$$

and, moreover, $p\left(A_{i}=x \mid a=k, b=m\right)=p\left(A_{i}=x, a=k, b=\right.$ $m) / p(a=k, b=m)=p\left(A_{i}=x, a=k\right) / p(a=k)$. Thus

$$
p\left(A_{i}=x \mid a=k, b=m\right)=p\left(A_{i}=x \mid a=k\right) .
$$

Thus, for measurement of a single observable, conditioning by the "two-side selection" of experimental settings is equivalent to the conditioning by the "one-side selection."

We also have:

$$
\begin{gathered}
p\left(A_{i}=0, B_{j}=0\right)=p\left(A_{i}=0, B_{j}=0, a \neq i, b \neq j\right) \\
=p\left(A_{i}=0, B_{j}=0, a \neq i, b \neq j\right) \\
+p\left(A_{i}=0, B_{j}=1, a \neq i, b \neq j\right)+p\left(A_{i}=0, B_{j}=-1, a \neq i, b \neq j\right)
\end{gathered}
$$




$$
\begin{aligned}
=p\left(A_{i}\right. & =0, a \neq i, b \neq j)=p\left(A_{i}=0, a \neq i\right) p(b \neq j) \\
& =p\left(A_{i}=0 \mid a \neq i\right) p(a \neq j) p(b \neq j) .
\end{aligned}
$$

By using $\mathbf{C 1}$ we obtain

$$
p\left(A_{i}=0, B_{j}=0\right)=p(a \neq j) p(b \neq j)=p(a \neq j, b \neq j) .
$$

Thus the probability of the event "no detection for the pair of observables $\left(A_{i}, B_{j}\right)$ " is equal to the probability of the event "the pair of random generators did not take the values $(i, j)$ ". From (51) and (6) we find that events of nondetection in the A-device and the B-device are independent:

$$
p\left(A_{i}=0, B_{j}=0\right)=p\left(A_{i}=0\right) p\left(B_{j}=0\right) .
$$

This is one of signs of "locality" of the probabilistic model. In fact, even detection in one of devices is independent from nondetection at another, e.g., for $\epsilon= \pm 1$,

$$
\begin{gathered}
p\left(A_{i}=\epsilon, B_{j}=0\right)=p\left(A_{i}=\epsilon, B_{j}=0, a=i, b \neq j\right) \\
=p\left(A_{i}=\epsilon, B_{j}=0, a=i, b \neq j\right) \\
+p\left(A_{i}=\epsilon, B_{j}=1, a=i, b \neq j\right)+p\left(A_{i}=\epsilon, B_{j}=-1, a=i, b \neq j\right) \\
=p\left(A_{i}=\epsilon, a=i, b \neq j\right)=p\left(A_{i}=\epsilon, a=i\right) p(b \neq j) \\
=p\left(A_{i}=\epsilon \mid a=i\right) p(a=i) p(b \neq j) .
\end{gathered}
$$

Finally, we use (5) and (8):

$$
p\left(A_{i}=\epsilon, B_{j}=0\right)=p\left(A_{i}=\epsilon\right) p\left(B_{j}=0\right) .
$$

The same is valid for the $B$-detection:

$$
p\left(A_{i}=0, B_{j}=\epsilon^{\prime}\right)=p\left(A_{i}=0\right) p\left(B_{j}=\epsilon^{\prime}\right) .
$$

In terms of Bell's argument these are also the signs of "locality" of the model.

\section{Classical probabilistic description of no-signaling}

In the Kolmogorov model the probabilities for the values of a single random variable can be reconstructed from the probabilities for the 
values of pairs of random variables. For our model, this implies that, for $j=1,2$,

$$
p\left(A_{i}=x\right)=\sum_{y} p\left(A_{i}=x, B_{j}=y\right),
$$

where $x, y=0, \pm 1$. This is an evident feature of a probability measure, we call it the condition of marginal consistency.

In quantum mechanics the no-signaling condition is realization of the condition of marginal consistency in context of the Bell-type tests, see the work of Kofler and Brukner [48] for detailed analysis of the problem in the rigorous probabilistic framework. As was pointed out in section 3.1, in our classical probabilistic model "quantum probabilities" appear as conditional probabilities (conditioning with respect to fixed experimental settings). In general the condition of marginal consistency for "absolute probabilities", see (27), does not coincide with this condition for conditional probabilities. The latter can be violated. So, in general "signaling" is possible (of course, here we discuss, as is common in the Bell framework, (no)signaling in purely probabilistic terms, see remark at the beginning of section 5). However, we shall show that under the conditions of (probabilistic) locality, LO and LIOG, the "absolute marginal consistency" (27) implies the conditional marginal consistency:

$$
p\left(A_{i}=\epsilon \mid a=i\right)=\sum_{\epsilon^{\prime}} p\left(A_{i}=\epsilon, B_{j}=\epsilon^{\prime} \mid a=i, b=j\right) .
$$

We prove this statement. The equality (11) implies

$$
\Sigma=\sum_{\epsilon^{\prime}} p\left(A_{i}=\epsilon, B_{j}=\epsilon^{\prime} \mid a=i, b=j\right)=\frac{1}{p(a=i, b=j)} \sum_{\epsilon^{\prime}} p\left(A_{i}=\epsilon, B_{j}=\epsilon^{\prime}\right) .
$$

For "absolute probabilities" we have

$$
\sum_{\epsilon^{\prime}} p\left(A_{i}=\epsilon, B_{j}=\epsilon^{\prime}\right)=p\left(A_{i}=\epsilon\right)-p\left(A_{i}=\epsilon, B_{j}=0\right) .
$$

Independence of detection in the $A$-device from nondetection in the $B$-device 2 , see (26), implies:

$$
\Sigma=\frac{1}{p(a=i, b=j)}\left(p\left(A_{i}=\epsilon\right)-p\left(A_{i}=\epsilon, B_{j}=0\right)\right)=\frac{p\left(A_{i}=\epsilon\right)\left(1-p\left(B_{j}=0\right)\right)}{p(a=i, b=j)} .
$$

Finally, we use (6), independence of random generators, and (8):

$$
\Sigma=\frac{p\left(A_{i}=\epsilon\right)(1-p(b \neq j))}{p(a=i, b=j)}=\frac{\left.p\left(A_{i}=\epsilon\right) p(b=j)\right)}{p(a=i) p(b=j)}=p\left(A_{i}=\epsilon \mid a=i\right) .
$$

\footnotetext{
${ }^{2}$ The terminology might be misleading. To escape this problem, we recall that in our model detectors have 100\% efficiency; nondetection means just that this experimental setting was not selected.
} 
We remark that the condition of marginal consistency is well known in mathematical psychology under the name of marginal selectivity, e.g., Dzhafarov et al. [49], [50]; see Asano et al. [51] recent studies.

\section{Classical probabilistic viewpoint on violation of the CHSH-inequality}

In this section we shall use the special symbol for the conditional probabilities corresponding to quantum probabilities, we set

$$
\begin{gathered}
q\left(A_{i}=\epsilon\right) \equiv p\left(A_{i}=\epsilon \mid a=i\right), q\left(B_{j}=\epsilon^{\prime}\right) \equiv p\left(B_{j}=\epsilon^{\prime} \mid b=j\right), \\
q\left(A_{i}=\epsilon, B_{j}=\epsilon^{\prime}\right) \equiv p\left(A_{i}=\epsilon \mid a=i, b=j\right) .
\end{gathered}
$$

On one hand, we know that for classical probabilities the CHSHinequality holds; on the other hand, we know that for quantum probabilities it can be violated. Consider classical correlations $(i, j=1,2)$ :

$$
\begin{gathered}
C_{i j}=E A_{i} B_{j}=p\left(A_{i}=+1, B_{j}=+1\right)+p\left(A_{i}=-1, B_{j}=-1\right) \\
-p\left(A_{i}=+1, B_{j}=-1\right)-p\left(A_{i}=-1, B_{j}=+1\right) .
\end{gathered}
$$

They are simply connected with quantum correlations:

$Q_{i j}=E\left[A_{i} B_{j} \mid a=i, b=j\right]=\frac{C_{i j}}{p(a=i, b=j)}$ or $C_{i j}=p(a=i, b=j) Q_{i j}$.

The CHSH-combination of correlations is given by the quantity:

$$
S=C_{11}+C_{12}+C_{21}-C_{22}
$$

It satisfies the CHSH-inequality (which is simple theorem in the Kolmogorov probability theory):

$$
|S| \leq 2
$$

We now write this inequality in term od quantum probabilities (classical conditional probabilities):

$\left|Q_{11} p(a=1, b=1)+Q_{12} p(a=1, b=2)+Q_{21} p(a=2, b=1)-Q_{22} p(a=2, b=2)\right| \leq 2$.

Such weighted CHSH-inequality cannot be violated even by quantum correlations - in contrast to the standard (unweighted) CHSHinequality which is typically used for quantum correlations:

$$
\left|Q_{11}+Q_{12}+Q_{21}-Q_{22}\right| \leq 2,
$$


but, as we have seen, its use is unjustified.

For example, let $p(a=i, b=j)=1 / 4$. Then (301) takes the form:

$$
\left|Q_{11}+Q_{12}+Q_{21}-Q_{22}\right| \leq 8 .
$$

But the number in the right-hand side, rhs $=8$, is too large. Since each quantum correlation $Q_{i j}$ is less than 1 (because even each conditional probability is probability, i.e., it is normalized by 1 , and each of random variables $A_{i}, B_{j}$ takes the values in $\left.[-1,+1]\right)$, one can expect at least $\mathrm{rhs}=4$.

The main point is that the CHSH inequality holds for an arbitrary Kolmogorov space and an arbitrary quadrupole of random variables taking values in $[-1,+1]$. However, our model is based on special quadrupole of random variables. We can use this specialty. It can easily shown that for the "absolute Kolmogorov correlations $C_{i j}$ a stronger inequality holds true:

$$
|S| \leq 1
$$

For $p(a=i, b=j)=1 / 4$, it implies that

$$
\left|Q_{11}+Q_{12}+Q_{21}-Q_{22}\right| \leq 4 .
$$

\section{Conclusions}

We demonstrated that "quantum probabilities and correlations" can be peacefully embedded in the classical Kolmogorov model: they have to be interpreted as conditional probabilities, where conditioning is with respect to fixed experimental settings. This approach leads to the weighted CHSH-inequality (34) (for symmetric and independent random generators determining settings of measurement devices) and not to the standard CHSH-inequality (31). In the classical probabilistic framework the violation of the latter can be expected. Of course, our scheme based on the complete account of randomness can be applied to any experiment in which a few (in general incompatible) experimental settings are involved. Therefore one can expect violation of (31) for conditional probabilities and the corresponding conditional correlations arising in various experiments, i.e., not only the experiments of the EPR-Bohm-Bell type.

Therefore "quantumness" is characterized not by a violation of the inequality (31), i.e., not by the experimentally confirmed fact that $\left|Q_{11}+Q_{12}+Q_{21}-Q_{22}\right|$ can be larger than 2, but by the Tsirelson bound $2 \sqrt{2}$. It cannot be explained in the classical Kolmogorov framework. 
We also emphasize that our classical probabilistic model does not provide the objective representation of observables; although one can assign simultaneously the values for both observables $A_{1}, A_{2} 2$ as well as for $B_{1}, B_{2}$ (as in any classical probabilistic model), the probability to obtain a nontrivial combination, $\left(\epsilon_{1}, \epsilon_{2}\right), \epsilon_{i}= \pm 1$, equals to zero. Thus the possibility of the classical probabilistic description is not equivalent to the possibility to treat observables as objective entities, cf. Zeilinger [52], 553] and Brukner and Zeilinger [54]-[56]. In general our model as well as the quantum model is not about "reality as it is", but about information which can be gained with the aid of observables, cf. again with as well as works of Chiribella, DAriano, and Perinotti [57] on the information approach to QM.

Although our model cannot be considering as supporting the ideas about hidden variables and in general it matches well with the information interpretation of QM, in the light of the existence of the classical probabilistic model for quantum probabilistic data violating the CHSH-inequality the problem of irreducible quantum randomness (from von Neumann to Brukner and Zeilinger) has to be reanalyzed once again. Since the quantum probabilities and correlations can be represented as the classical entities (conditional with respect to the selections of experimental contexts), one has to understand how conditioning can shadow the irreducible randomness. It seems that here the key word is contextuality. Everybody would agree that there is a link (not completely understandable) between contextuality and quantum randomness. The usage of the specialty of quantum randomness in some schemes of quantum cryptography and theory of quantum random generators lifts the problem of the coexistence of quantum randomness and classical probabilistic representation to the level of quantum technologies.

\section{acknowledgments}

This paper was written during author's visiting professor fellowship to the Institute for Quantum Optics and Quantum Information of Austrian Academy of Science (April-June, 2014); the main result of this paper was presented in the course of lectures on the inter-relation between classical and quantum randomness given for the graduate students of this institute. I would like to thank Anton Zeilinger for hospitality and critical discussions about the objective representation of quantum observables and the possibility to apply the mathematical formalism of quantum mechanics outside of quantum physics. 


\section{References}

[1] Bell J S 1964 On the Einstein Podolsky Rosen Paradox, Physics $1,3,195200$

[2] Bell J S 1987 Speakable and Unspeakable in Quantum Mechanics (Cambridge: Cambridge Univ. Press)

[3] Khrennikov A (ed) 2001 Foundations of Probability and Physics. Quantum Probability and White Noise Analysis vol 13 (Singapore: WSP)

[4] Khrennikov A (ed) 2002 Quantum Theory: Reconsideration of Foundations. Ser. Math. Modelling vol 2 (Växjö: Växjö Univ. Press)

[5] Khrennikov A (ed) 2003 Quantum Theory: Reconsideration of Foundations-2. Ser. Math. Modelling vol 10 (Växjö: Växjö Univ. Press)

[6] Khrennikov A (ed) 2005 Foundations of Probability and Physics3. vol 750

[7] G. Adenier, C. Fuchs, and A. Khrennikov (eds) 2007 Foundations of Probability and Physics-4, American Institute of Physics, Ser. Conference Proceedings, 889, Melville, NY.

[8] Adenier, G., Khrennikov, A. Yu., Lahti, P., Manko, V. I., and Nieuwenhuizen, Th.M. (eds) 2008 Quantum Theory: Reconsideration of Foundations-4, American Institute of Physics, Ser. Conference Proceedings 962, Melville, NY

[9] L. Accardi, G. Adenier, C.A. Fuchs, G. Jaeger, A. Khrennikov, J.-A. Larsson, S. Stenholm (eds) 2009 Foundations of Probability and Physics-5, American Institute of Physics, Ser. Conference Proceedings, 1101, Melville, NY.

[10] Khrennikov A (ed) 2010 Quantum Theory: Reconsideration of Foundations-5 vol 1232 (Melville, NY: AIP)

[11] Jaeger G, Khrennikov A, Schlosshauer M, Weihs G (eds) 2011 Advances in Quantum Theory vol 1327 (Melville, NY: AIP)

[12] Giustina M, Mech Al, Ramelow S, Wittmann B, Kofler J, Beyer J, Lita A, Calkins B, Gerrits Th, Woo Nam S, Ursin R, and Zeilinger A 2013 Nature 497227

[13] Christensen B G et al. 2013 Phys. Rev. Lett. 1111304

[14] A. Khrennikov, S. Ramelow, R. Ursin, B. Wittmann, J. Kofler, and I. Basieva, On the equivalence of the Clauser-Horne and Eberhard inequality based tests, Physica Scripta (to be published). 
[15] Kolmogoroff A N 1933 Grundbegriffe der Wahrscheinlichkeitsrechnung ( Berlin: Springer Verlag); English translation: Kolmogorov A N 1956 Foundations of Theory of Probability (New York: Chelsea Publishing Company)

[16] L. Accardi 1981 Topics in quantum probability. Phys. Rep. 77 169-192.

[17] Kupczynski M 1987 Phys. Lett. A 121205

[18] A. Khrennikov, Interpretations of Probability. De Gruyter, Berlin, 2009, second edition (completed); first edition 1998.

[19] L. Accardi, M. Regoli, Locality and Bell's inequality. arXiv:quant-ph/0007005.

[20] De Muynck W M 2002 Foundations of Quantum Mechanics, an Empiricists Approach (Dordrecht: Kluwer)

[21] K. Hess and W. Philipp 2002 Europhys. Lett. 57, 775 (2002).

[22] Kupczynski M 2002 On the Completeness of Quantum Mechanics, arXiv:quant-ph/028061

[23] Accardi L 2005 Some loopholes to save quantum nonlocality. Foundations of Probability and Physics-3 vol 750 (Melville, NY: AIP) pp 1-20

[24] Kupczynski M 2005 J. Russian Laser Research 26514

[25] Kupczynski M Seventy Years of the EPR Paradox. Albert Einstein Century Conference vol 861 (Melville, NY: AIP) pp 516523

[26] Adenier G. 2008 Am. J. Phys. 76147

[27] A. Khrennikov, Contextual approach to quantum formalism, Springer, Berlin-Heidelberg-New York, 2009.

[28] Adenier G 2008 Local Realist Approach and Numerical Simulation of Nonclassical Experiments in Quantum Mechanics ( V̈̈jö: V ẍjö Univ. Press)

[29] Khrennikov A 2008 Entropy 1019

[30] De Raedt H, De Raedt K, Michielsen K, Keimpema K, and Miyashita S 2007 Phys. Soc. Japan 76104005

[31] Hess K, Michielsen K and De Raedt H 2009 EPL 8760007

[32] Fine A 1989 Found. Phys. 19453

[33] Clauser, J. F., Horne, M. A., Shimony, A., and Holt, R. A. Phys. Rev. Lett. 23, 880-884 (1969). 
[34] Avis D, Fischer P, Hilbert A, and Khrennikov A 2009 Single, Complete, Probability Spaces Consistent With EPR-Bohm-Bell Experimental Data, Foundations of Probability and Physics-5 vol 750 (Melville, NY: AIP) pp 294-301.

[35] A. Khrennikov 2014 Classical probability model for Bell inequality. EmQM13: Emergent Quantum Mechanics36 October 2013, Vienna, Austria. J. Phys.: Conf. Ser., 504.

[36] A. Khrennikov, Ubiquitous quantum structure: from psychology to finances, Springer, Berlin-Heidelberg-New York, 2010.

[37] Dzhafarov, E.N., and Kujala, J.V. (2014). Embedding quantum into classical: contextualization vs conditionalization. PLoS ONE 9(3): e92818. doi:10.1371/journal.pone.0092818.

[38] Dzhafarov, E.N., and Kujala, J.V. (2014). No-Forcing and NoMatching theorems for classical probability applied to quantum mechanics. Foundations of Physics 44, 248-265.

[39] J. Kofler, T. Paterek, and C. Brukner, Experimenter's freedom in Bell's theorem and quantum cryptography. Phys. Rev. A 73, 022104 (2006).

[40] M. A. De Gosson (2002) The "symplectic camel principle" and semiclassical mechanics. J. Physics A: Mathematical and General 35 (32), 6825

[41] M. A. De Gosson (2006) The Uncertainty Principle. Symplectic Geometry and Quantum Mechanics, 237-269.

[42] V. I. Man'ko (1996) Classical formulation of quantum mechanics. J. Russian Laser Research 17, N 6, 579-584.

[43] S. Mancini, V. I. Man'ko, P. Tombest (1997) Classical-like description of quantum dynamics by means of symplectic tomography. Foundations of Physics 27, 801-824.

[44] M. D'Ariano, Probabilistic theories: What is special about quantum mechanics? In: Philosophy of Quantum Information and Entanglement, Al. Bokulich, G. Jaeger (eds), Cambridge University press, Cambridge, 2010, pp. 85-110.

[45] A. Khrennikov, B. Nilsson, S. Nordebo, and I. Volovich. Photon flux and distance from the source: consequences for quantum communication Foundations of physics, (DOI) 10.1007/s10701014-9786-0

[46] T. Ishiwatari, A. Khrennikov, B. Nilsson, and I. Volovich. Quantum field theory and distance effects for polarization correlations in waveguides. In 3rd Conf. Mathematical Modeling of 
Wave Phenomena/20th Nordic Conference on Radio Science and Communications, volume 1106 of AIP Conference Proceeding, pages 276-285. American Inst. of Physics, 2009.

[47] A. Khrennikov, B. Nilsson, S. Nordebo, and I. Volovich. Distance dependence of entangled photons in waveguides. In Conference FPP6-Foundations of Probability and Physics-6, volume 1424 of AIP Conference Proceeding, pages 262-269, Melville, New York, 2012. American Inst. of Physics.

[48] J. Kofler, C. Brukner (2013) Condition for macroscopic realism beyond the Leggett-Garg inequalities. Phys. Rev. A 87, 052115.

[49] Dzhafarov, E.N. (2003). Selective influence through conditional independence. Psychometrika, 68, 7-26.

[50] Dzhafarov, E.N., and Kujala, J.V. (2014). On selective influences, marginal selectivity, and Bell/CHSH inequalities. Topics in Cognitive Science 6, 121128.

[51] M. Asano, T. Hashimoto, A. Khrennikov, M. Ohya, Y. Tanaka Violation of contextual generalization of the Leggett-Garg inequality for recognition of ambiguous figures. arXiv:1401.2897 [q-bio.NC]

[52] A. Zeilinger. A foundational principle for quantum mechanics. Foundations of Physics, 29(4):631-643, 1999.

[53] A. Zeilinger, Dance of the Photons: From Einstein to Quantum Teleportation. Farrar, Straus and Giroux, New-York, (2010)

[54] C. Brukner and A. Zeilinger, Malus' law and quantum information. Acta Physica Slovava, 49(4):647-652, 1999.

[55] C. Brukner and A. Zeilinger, Operationally invariant information in quantum mechanics. Physical Review Letters, 83(17):3354-3357, 1999.

[56] C. Brukner and A. Zeilinger, Information Invariance and Quantum Probabilities, Found. Phys. 39, 677 (2009).

[57] G. Chiribella, G. M. DAriano, and P. Perinotti Informational derivation of quantum theory. Phys. Rev. A 84, 012311 (2011). 